\title{
Extracting real-crack properties from non-linear elastic behaviour of rocks: abundance of cracks with dominating normal compliance and rocks with negative Poisson ratios
}

\author{
Vladimir Y. Zaitsev ${ }^{1}$, Andrey V. Radostin ${ }^{1}$, Elena Pasternak ${ }^{2}$, and Arcady Dyskin ${ }^{3}$ \\ ${ }^{1}$ Institute of Applied Physics, Russian Academy of Sciences, Nizhny Novgorod, 603950, Russia \\ ${ }^{2}$ School of Mechanical and Chemical Engineering, The University of Western Australia, Perth, 6009, Australia \\ ${ }^{3}$ School of Civil, Environmental and Mining Engineering, The University of Western Australia, Perth, 6009, Australia \\ Correspondence to: Vladimir Y. Zaitsev (vyuzai@ipfran.ru)
}

Received: 12 February 2017 - Discussion started: 17 March 2017

Revised: 7 July 2017 - Accepted: 7 July 2017 - Published: 5 September 2017

\begin{abstract}
Results of examination of experimental data on non-linear elasticity of rocks using experimentally determined pressure dependences of $\mathrm{P}$ - and S-wave velocities from various literature sources are presented. Overall, over 90 rock samples are considered. Interpretation of the data is performed using an effective-medium description in which cracks are considered as compliant defects with explicitly introduced shear and normal compliances without specifying a particular crack model with an a priori given ratio of the compliances. Comparison with the experimental data indicated abundance $(\sim 80 \%)$ of cracks with the normal-toshear compliance ratios that significantly exceed the values typical of conventionally used crack models (such as penny-shaped cuts or thin ellipsoidal cracks). Correspondingly, rocks with such cracks demonstrate a strongly decreased Poisson ratio including a significant $(\sim 45 \%)$ portion of rocks exhibiting negative Poisson ratios at lower pressures, for which the concentration of not yet closed cracks is maximal. The obtained results indicate the necessity for further development of crack models to account for the revealed numerous examples of cracks with strong domination of normal compliance. Discovering such a significant number of naturally auxetic rocks is in contrast to the conventional viewpoint that occurrence of a negative Poisson ratio is an exotic fact that is mostly discussed for artificial structures.
\end{abstract}

\section{Introduction}

It is widely appreciated that most rocks exhibit strongly increased tensosensitivity, that is, giant elastic non-linearity as compared with atomic non-linearity of homogeneous solids and liquids. A bright manifestation of this non-linearity is a very pronounced dependence of rocks' elastic moduli on applied pressure. The main reason for this giant non-linearity is the presence of highly compliant cracks and contacts in the relatively hard matrix.

Important features of this "soft-hard paradigm" of giant non-linearity in microstructured solids (Guyer and Johnson, 2009; Zaitsev et al., 2009a) can be explained by very instructive and simple 1-D rheological models in which highly compliant cracks/contacts correspond to soft elastic elements/springs contained in a relatively hard matrix (Zaitsev, 1996; Belyaeva and Zaitsev, 1997, 1998). Such models can be very useful for elucidating why the relationship between concentration of the soft inclusions and the resultant non-linearity level can be non-monotonic. Also, they can provide some understanding of the origin of frequency dependence of such microstructure-induced non-linearity as an influence of relaxation localized at the same soft defects. Furthermore, those rheological models clearly demonstrate that relaxation properties of the soft defects in addition to the elastic non-linearity (i.e. tensosensitivity of elastic moduli) inevitably lead to pronounced tensosensitivity of dissipation in microstructured solids (Zaitsev and Matveev, 2006; Zaitsev et al., 2008; Nazarov and Radostin, 2015) that may exhibit itself as dissipative non-linearity (which agrees with 
considerations based on physical crack models Fillinger et al., 2006; Zaitsev et al., 2011; Zaitsev and Matveev, 2012).

Despite the usefulness of the above-mentioned 1-D models for understanding basic features of the influence of highly compliant inclusions on reduction of the elastic modulus and the origin of its giant stress sensitivity, closer comparison with seismo-acoustic properties of real rocks requires the effective-medium models that more adequately correspond to the 3-D character of real rocks. Even in the simplest isotropic approximation, rocks are characterized by a pair of independent elastic moduli. The most widely used are the bulk modulus, the shear modulus determining the velocity of shear $\mathrm{S}$-waves, the Young modulus, as well as the modulus corresponding to the velocity of longitudinal P-waves. Among those moduli any two ones can be considered independent and the others are expressed via the chosen pair of independent ones.

Since cracks are the simplest and most important type of compliant defect in consolidated rocks, considerable attention was paid to developing models that describe crackinduced variations in elastic moduli. Although such descriptions differ in the way of accounting for eventual interaction of cracks (i.e. small perturbation or approximation of low crack concentrations, without accounting for mutual crack interaction (Walsh, 1965), the so-called self-consistent approach (O'Connell and Budiansky, 1974), or the differential approach (Zimmerman, 1985)), the representations of cracks in such models were based on the same geometries for which exact expressions were available that describe the stored elastic energy in the presence of shear stress or stress normally directed to the crack plane. In particular, the so-called pennyshaped cracks or thin elliptical voids with small aspect ratios have been widely used.

Despite the differences in the methods accounting for interaction of cracks at larger concentrations, in the limiting case of small crack concentrations all such models predict identical complementary variations for the chosen independent elastic moduli. For example, the chosen crack geometry pre-determines a given very specific proportion between variations in the $\mathrm{S}$ - and $\mathrm{P}$-wave velocities under hydrostatic pressure. Observations for real rocks, however, often demonstrate different proportions between crack-induced variations in the $\mathrm{P}$ - and $\mathrm{S}$-wave velocity variations, such that playing with crack concentrations in the above-mentioned models in principle cannot help to reach better agreement between the predictions and observations.

The fact that variations of different moduli inferred from the measured wave velocities require different crack concentrations implies that real cracks could be characterized by significantly different proportions between their shear and normal compliances. Such variability of crack properties in principle cannot be accounted for in conventional effectivemedium models based on cracks modelled as straight cuts of any geometry (e.g. penny-shaped) or thin ellipsoidal voids with a small aspect ratio. In such conventionally used models the ratio between those compliances is pre-determined and cannot exhibit significant variations.

This fact motivated the development of alternative effective-medium models in which cracks are considered as highly compliant defects with independently defined normal and shear compliance without a predetermined proportion between them. Such an idea was realized in Zaitsev and Sas (2000) and equivalent expressions (that differ only by a normalization) were derived in MacBeth (2004) based on results of Sayers and Kachanov (1995). Using results from Zaitsev and Sas (2000), in Zaitsev and Sas (2004) from the analysis of pressure dependences of two elasticwave velocities in three samples, ratios between normal and shear compliances were extracted in the approximation of non-interacting cracks and in Zaitsev et al. (2017) possible crack interaction was accounted for using the differential scheme. For one of the examined samples, the inferred crack characteristics did not differ strongly from the ones of penny-shaped cracks, whereas the other two demonstrated 24 times stronger dominance of normal compliance of the real defects. Furthermore, one of the samples (Weber sandstone studied in Coyner, 1984) with the highest normal-to-shear compliance ratio of the cracks was found to possess negative Poisson ratios at lower confining pressures (up to $20 \mathrm{MPa}$ ). With increasing pressure (that caused gradual closing of the cracks) the Poisson ratio gradually increased towards to "normal" positive values.

Results from Zaitsev and Sas (2004) already demonstrated that real-crack properties may significantly differ from those implied in the popular model of penny-shaped cracks, which agrees with some recent works (Gurevich et al., 2009; Sayers and Han, 2002) where some other facts indicating insufficiency of models based on penny-shaped cracks were also discussed. However, the fairly small number of rocks discussed in those papers did not yet allow one to estimate how exotic are samples inconsistent with the penny-shaped cracks. In what follows, based on examination of pressure dependences for $\sim 90$ rocks (Freund, 1992; Mavko and Jizba, 1994; Han, 1986), we present results demonstrating that the "unusual" properties of real cracks are quite common. Furthermore, we show that the relevance of the penny-shaped crack concept can be rather considered as an exception, and rocks with negative Poisson ratios are not rare exceptions, in contrast to conventional belief. Reliable reconstruction of compliance properties of cracks (that are conventionally used in models of linear elastic properties of rocks) in fact requires consideration of non-linear behaviour of rocks - pressureinduced variation of their elastic properties in a sufficiently wide pressure range. In the course of this consideration we will also point out some aspects of rock non-linearity (tensosensitivity) that have not been explicitly discussed earlier. 


\section{Non-linear variation of rock elasticity under varying pressure: implications of 1-D modelling}

In geophysics elastic non-linearity of rocks is well appreciated; however, when considering non-linear propagation of elastic waves, the modelling is often simplified by using 1$\mathrm{D}$ approximation starting from a 1-D constituent non-linear stress-strain relationship in which non-linearity is often considered quadratic in strain. For the present consideration of non-linear variations of elastic moduli under isotropic hydrostatic compression that affects the state high compliant defects, 1-D description can also be used, for example, in the form with small (quadratic in strain) non-linear correction to the linear stress-stress relation:

$\sigma(\varepsilon)=\varepsilon \cdot M\left\{1+\varepsilon \cdot \gamma^{(2)}\right\}=\varepsilon \cdot M+\gamma^{(2)} \cdot M \cdot \varepsilon^{2}$.

Here $M$ is the linear elastic modulus of the medium and $\gamma^{(2)}$ is its dimensionless non-linearity parameter characterizing the deviation of stress created in the medium from the linear proportionality to strain. Strictly speaking, strain is defined with respect to some initial state of the medium, so that the local slope of the dependence around a current degree of deformation becomes dependent on strain (and thus stress) and can be considered as a current elastic modulus:

$M(\varepsilon)=\partial \sigma / \partial \varepsilon$.

It is clear that the dimensionless parameter of the quadratic non-linearity can be expressed as

$\gamma^{(2)}=\frac{1}{2 \cdot M} \partial M(\varepsilon) / \partial \varepsilon=\frac{1}{2} \frac{d M}{d \sigma}$

taking into account that the stress (pressure) increment is related to the strain increment as $d \sigma=M \cdot d \varepsilon$.

In contrast to homogeneous materials with weak atomic non-linearity and the non-linearity parameter of the order of unity (Zarembo and Krasilnikov, 1971), in heterogeneous media their non-linearity can be strongly increased due to the presence of highly compliant defects with a strongly locally decreased elastic modulus. Due to this fact the strain becomes strongly locally increased at the soft defects, which results in strong enhancement of their local non-linear deviation from the linear stress-strain law and, correspondingly, leads to enhancement of the average (macroscopic) non-linearity of the material.

Important features of the microstructure-induced nonlinearity can be revealed in the framework of the abovementioned 1-D description (Zaitsev, 1996; Belyaeva and Zaitsev, 1997). The simplest for understanding is the case of identical compliant defects: if the relative volume content (concentration) of such highly compliant defects is $v$ and the defects are of the same type, the effective quadratic nonlinearity parameter becomes strongly increased:

$\gamma^{(2)} / \gamma_{0}^{(2)} \approx\left(1+v / \varsigma^{2}\right) /(1+v / \varsigma)^{2}$, where the small parameter, $\varsigma \ll 1$, characterizes the relative compliance of the compliant defects with respect to the homogeneous matrix material; parameter $\gamma_{0}^{(2)} \sim 1$ characterizes its own weak non-linearity of the material of the defects. A clear example is a liquid with gas bubbles: taken separately the liquid and gas both are weakly non-linear, but the non-linearity of the mixture may become giant. For a sufficiently small compliance parameter $\varsigma \ll 1$ (that may be $10^{-3}-10^{-4}$ like for a gas-water mixture), even if the concentration $v$ is small, the non-linearity parameter can exhibit a giant increase, $\gamma^{(2)} / \gamma_{0}^{(2)} \gg 1$, because the combination $v / \varsigma^{2}$ in Eq. (4) may become large.

Simultaneously with increase in the non-linearity parameter, the elastic modulus $M$ due to the presence of high compliant defects exhibits gradual decrease in comparison with modulus $M_{0}$ of the homogeneous matrix:

$M / M_{0} \approx 1 /(1+v / \varsigma)$.

Comparing Eqs. (3) and (4) one can easily notice that even if the decrease in the elastic modulus is yet small, $v / \varsigma \ll 1$, the increase in the non-linearity parameter, Eq. (4), may become very significant $\left(\gamma^{(2)} / \gamma^{(2)} \gg 1\right)$. Indeed, even if in Eq. (5) $v / \varsigma \ll 1$, the combination $v / \varsigma^{2}$ in Eq. (4) may become large for $\varsigma \ll 1$. Furthermore, the non-linearity parameter reaches its maximum value $\sim 1 / \varsigma$ for a rather small concentration of the defects $v=\varsigma$, for which the elastic modulus becomes twice decreased and the interplay between the local strain enhancement and the concentration of the defects is optimal (Zaitsev, 1996; Belyaeva and Zaitsev, 1997).

In contrast to the above-mentioned bubbly liquids, for which the bubbles have the same contrast $\varsigma$ in compressibility with the liquid and the existence of a clear maximum of the non-linear parameter in its dependence on bubble concentration is a known fact (Trivett et al., 2006; Zaitsev et al., 2009b), for cracked rocks, the fact of maximal non-linearity at an intermediate concentration of cracks is not typical. Bearing in mind that for the bulk modulus $K$ of rocks under hydrostatic compression, the 1-D description is applicable as far as the normal compliance of cracks is concerned (see below for more details); we note that pressure dependences of $K(P)$ usually demonstrate an ever-increasing slope $d K(P) / d P$ (i.e. the non-linearity parameter) with decreasing confining pressures $P$ at which the concentration cracks that are not closed gradually increase. Typical examples of $K(P)$ recalculated from experimentally measured $\mathrm{P}$ - and $\mathrm{S}$-wave velocities are shown in Fig. 1a for several sandstone samples that are often discussed in the literature (Coyner, 1984; Mavko and Jizba, 1994).

This gradual increase in slope $d K / d P$ with decreasing pressure is quite naturally attributed to a broad distribution of the compliant defects over their compliance parameter. Indeed, it is widely accepted that with increasing confining pressure the compliant crack-like defects gradually become tightly closed (starting from the most compliant) and 

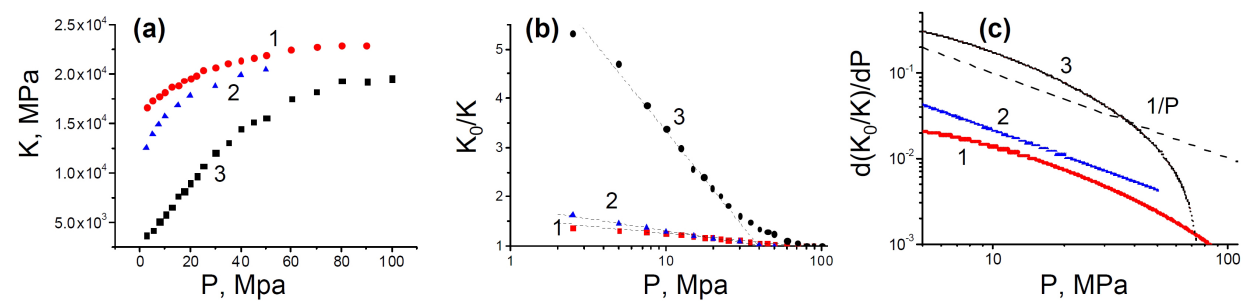

Figure 1. Non-linearity exhibited by dry Navajo, Nugget, and Weber sandstones (Coyner, 1984; Mavko and Jizba, 1994). (a) Typical dependences $K(P)$ recovered from the experimentally measured pressure dependences of P- and S-wave velocities; (b) the same data represented as the pressure dependences for normalized inverse bulk modulus $K_{0} / K(P)$ shown in the plot with a logarithmic pressure axis; (c) derivatives $d\left(K_{0} / K\right) / d P=-(d K / d P) K_{0} / K^{2}$ calculated using the approximating curves (in the form of third-order polynomials). Numbers 1 , 2, and 3 denote the data for Navajo, Nugget, and Weber sandstones, respectively (Coyner, 1984; Mavko and Jizba, 1994; Han, 1986). The slopes of the approximating straight lines in panel (b) corresponding to the normalized Eq. (8) characterize the differences in the density of $\operatorname{cracks}\left(v_{0}^{(\varsigma)} \approx 0.13\right.$ for Navajo, $v_{0}^{(\varsigma)} \approx 0.21$ for Nugget, and $v_{0}^{(\varsigma)}=1.7$ for Weber). The dashed line in panel (c) shows the $1 / P$ dependence corresponding to the normalized Eq. (10). The deviation downwards of curve 3 for Weber in panel (c) is related to pronounced saturation of $K(P)$ and $K^{-1}(P)$ at higher pressures clearly visible in panels (a) and (b).

do not contribute anymore to the rock non-linearity. This agrees with known properties of narrow cracks with a small aspect ratio $\alpha \ll 1$, for which their aspect ratio determines the relative compliance $\alpha \sim \varsigma$. Such thin cracks are known to get closed under the average strain $\varepsilon_{\mathrm{c}} \sim \alpha$; the proportionality coefficient is of the order of unity and its value may differ somewhat, as demonstrated by solutions for elliptical cracks Morlier (1971), tapered non-elliptical cracks Mavko and Nur (1978), etc. Since the strains and applied pressure $P$ can be considered to be roughly proportional, $\varepsilon_{\mathrm{c}} \approx P_{\mathrm{c}} / K$, all these quantities can be considered to be approximately proportional to each other: $\varsigma \sim \alpha \sim \varepsilon_{\mathrm{c}} \sim P_{\mathrm{c}} / K$, which we take into account in the consideration below.

For non-identical defects leading to a distribution in the compliance parameter, Eqs. (3) and (4) should be modified to comprise the contributions of defects with different compliance parameters $\varsigma$ Belyaeva and Zaitsev (1998):

$K / K_{0} \approx 1 /\left(1+\int \frac{v(\varsigma)}{\varsigma} d \varsigma\right)$.

And the equation for the non-linear parameter can be rewritten as

$\frac{K_{0}}{K^{2}} \gamma^{(2)} / \gamma_{0}^{(2)} \approx 1+\int \frac{v(\varsigma)}{\varsigma^{2}} d \varsigma$

It is clear that, by analogy with Eqs. (4) and (5) for identical defects, the modulus reduction and the increase in nonlinearity are determined by the distribution $v(\varsigma)$ of the defect concentration over the compliance parameter $\zeta$.

If one consider ranges of pressure $P_{\min } \leq P \leq P_{\max }$ relevant to experiments (quite often this range is from several MPa to about $10^{2} \mathrm{MPa}$ ), i.e. with relative variation $P_{\max } / P_{\min } \sim 15-30$ times, as the examples in Fig. 1 show, the gradually closed/opened cracks should be distributed over the compliance parameter with a similar relative range, $\varsigma_{\max } / \varsigma_{\min } \sim P_{\max } / P_{\min }$. Since this range in the practically relevant case is not huge (not many orders of magnitude), one can assume that in the first approximation the function $v(\varsigma)$ may be approximated by a uniform distribution $v(\varsigma)=v_{0}^{(\varsigma)} \approx$ const. for $\varsigma_{\min } \leq \varsigma \leq \varsigma_{\max }$. Then one obtains

$$
\begin{aligned}
& K_{0} / K \approx 1+\int_{\varsigma}^{\varsigma_{\max }} \frac{v(\varsigma)}{\varsigma} d \varsigma=1+v_{0}^{(\varsigma)} \ln \left(\varsigma_{\max } / \varsigma\right) \\
& \approx 1+v_{0}^{(\varsigma)} \ln \left(P_{\max } / P\right), \\
& \frac{K_{0}}{K^{2}} \gamma^{(2)} / \gamma_{0}^{(2)} \approx 1+\int_{\varsigma}^{\varsigma_{\max }} \frac{v(\varsigma)}{\varsigma^{2}} d \varsigma=1-\frac{v_{0}^{(\varsigma)}}{\varsigma_{\max }}+\frac{v_{0}^{(\varsigma)}}{\varsigma} .
\end{aligned}
$$

Practically more useful than Eq. (9) can be a representation in the form of the direct derivative of Eq. (8), $d(1 / K) / d P=$ $-(d K / d P) / K^{2}$, that does not involve an unknown initial value of the non-linearity parameter. In view of relationship (8) it should be expected in the form

$$
\frac{K_{0}}{K^{2}} \frac{d K}{d P} \approx \frac{v_{0}^{(5)}}{P} .
$$

This dependence can be compared with experimental data. Figure $1 \mathrm{~b}$ shows the pressure dependences for the bulk modulus of the same samples as in Fig. 1a using a logarithmic scale of the pressure axis, for which the proportionality to $\log (P)$ should look like a straight line. It is clear that in Fig. $1 \mathrm{~b}$ such straight lines approximate the experimental dependences $K^{-1}(P)$ fairly well. The trends to saturation closer to maximal and minimal strains are expectable (since the distribution $v(\varsigma)=v_{0}^{(\varsigma)} \approx$ const. cannot be ideally flat). The slopes of the straight lines in Fig. $1 \mathrm{~b}$ are determined by $v_{0}^{(\varsigma)}$ and give a clear representation of the differences in the characteristic concentrations of the defects for the examined samples. Finally, Fig. 1c shows in double logarithmic scale the derivatives of the approximating curves shown in Fig. 1b, 
with a curve $1 / P$ as a guide for the eye. Thus Fig. $1 \mathrm{~b}$ and $c$ demonstrates that the simplest approximation of the distribution of the defects by a constant value reasonably agrees with the experimental observations in a fairly wide range of pressures $\left(P_{\max } / P_{\min } \sim 10-20\right.$ times $)$.

\section{Inferences from non-linear variations in elastic moduli of rocks in 3-D descriptions}

Now we recall that in the previous section we considered only 1-D descriptions that can be fairly well applied to the reduction in the bulk modulus under hydrostatic compression of real rock samples. However, in real 3-D rocks even under isotropic hydrostatic compression and fairly isotropically oriented cracks, there are two independent elastic moduli, of which usually the bulk modulus and shear modulus are considered. The crack-like defects with isotropic orientations can also be characterized by two independent compliances with respect to normal and shear loading. Using such a representation of cracks like planar defects with two compliances that are not a priori predetermined by a particular crack model, one can relate the values of different elastic moduli to the crack-effective densities and compliances by analogy with the above-considered 1-D case. Such expressions were obtained in Zaitsev and Sas (2000) in the form

$$
\begin{aligned}
& \widetilde{K}=\frac{K}{K_{0}}=\frac{1}{1+\frac{1}{3} N_{\mathrm{n}} /\left(1-2 \nu_{0}\right)}, \\
& \widetilde{G}=\frac{G}{G_{0}}=\frac{1}{1+\frac{2}{15} N_{\mathrm{n}} /\left(1+\nu_{0}\right)+\frac{2}{5} N_{\mathrm{s}}} .
\end{aligned}
$$

where by analogy with the above-considered 1-D case the subscript " 0 " denotes the matrix values, $N_{\mathrm{n}}=\int v(\varsigma) \varsigma^{-1} d \varsigma$ is the effective concentration of the normal compliance and $N_{\mathrm{s}}=\int v(\xi) \xi^{-1} d \xi$ is a similar quantity for the shear compliance, and $v_{0}$ is the Poisson ratio of the matrix rock. For the other moduli, one obtains similar expressions (Zaitsev and Sas, 2000). In these equations the shear compliance is normalized by the shear modulus of the rock matrix and the normal compliance is normalized by the Young modulus corresponding to the rock deformability under uniaxial stress. Instead of a single dimensionless compliance parameter used in the previous section (in fact corresponding to the normal compliance), these expressions contain two compliance parameters representing the normal and shear loading characterizing the defects. Factors $1 / 3,2 / 5$, etc. in Eqs. (11) and (12) are related to spatial averaging of isotropically oriented defects.
Similar equations were derived in MacBeth (2004) using basic relations obtained in Sayers and Kachanov (1995):

$$
\begin{aligned}
\widetilde{K} & =\frac{K}{K_{0}}=\frac{1}{1+K_{0} Z_{\mathrm{n}}}, \\
\widetilde{G} & =\frac{G}{G_{0}}=\frac{1}{1+\frac{4}{15} G_{0} Z_{\mathrm{n}}+\frac{2}{5} G_{0} Z_{\mathrm{s}}} .
\end{aligned}
$$

In these equations quantities $Z_{1}$ and $Z_{2}$ characterizing total normal and shear compliances imparted to the rock by cracks are dimensional (with the dimension of the inverse modulus). The shear compliance of the defects in both approaches is similarly compared with the shear elastic modulus of the matrix material. But the normal compliance in Eq. (12) is normalized differently: in Zaitsev and Sas (2000), Eqs. (11)(12), the normal compliance of the defects is compared with the Young modulus (i.e. the modulus that corresponds to uniaxial stress, so that the compliance parameter $\zeta$ of the defects with respect to normal uniaxial stress can be expressed as $\varsigma=E_{\text {crack }} / E_{0}$; the latter can be substituted in the expression for $\left.N_{\mathrm{n}}=\int v(\varsigma) \varsigma^{-1} d \varsigma\right)$. Then taking into account the conventional relationship $K=1 / 3 E /(1-2 v)$ between moduli $E$ and $K$ (Landau and Lifshitz, 1986), the combination $1 / 3 N_{\mathrm{n}} /\left(1-2 v_{0}\right)$ in Eq. (11) can be transformed into the form $1 / 3 N_{\mathrm{n}} /\left(1-2 v_{0}\right)=K_{0} Z_{\mathrm{n}}$ (where $\left.Z_{\mathrm{n}}=\int v(\varsigma) \varsigma^{-1} d \varsigma / E_{0}\right)$. As a result, Eq. (11) assumes the form of Eq. (13) in the notations of the paper by MacBeth (2004), where the normal compliance of the defects is normalized by the bulk modulus $K_{0}$ of the matrix. This comparison justifies Eqs. (11) and (13) for the effective bulk modulus having the same form as the 1-D Eq. (6) discussed in the previous section.

Note further that the total shear compliances $2 / 5 N_{\mathrm{s}}$ and $2 / 5 G_{0} Z_{\mathrm{s}}$ in Eqs. (12) and (14) have exactly the same meaning (coincide quantitatively). Then it can readily be verified that Eqs. (12) and (14) have exactly the same proportions between total normal and shear compliances: quantities $2 / 15 N_{\mathrm{n}} /\left(1+v_{0}\right)$ and $2 / 5 N_{\mathrm{s}}$ in Eq. (12) and quantities $4 / 15 G_{0} Z_{\mathrm{n}}$ and $2 / 5 G_{0} Z_{\mathrm{s}}$ in Eq. (14). Thus representation Eqs. (11), (12) and (13), (14) are equivalent and differ only by notation.

Assuming that both normal and shear compliances are localized at the same defects (like at penny-shaped cracks in conventional models), the ratio $q=N_{\mathrm{n}} / N_{\mathrm{s}}$ then characterizes the ratio between normal and shear compliances of the crack-like defects. Taking into account the difference in the normal-compliance normalization, one obtains $\widetilde{q}=Z_{\mathrm{n}} / Z_{\mathrm{s}}=1 / 2\left(N_{\mathrm{n}} / N_{\mathrm{s}}\right) /\left(1+v_{0}\right)$. Comparing Eqs. (11)(14) with expressions for elastic-moduli reduction based on the penny-shaped crack model (O'Connell and Budiansky, 1974; Zimmerman, 1985), one concludes that penny-shaped cracks correspond to the ratio of normal and shear compliances $q=\left(2-v_{0}\right)\left(1+v_{0}\right) \sim 2$ (if the normal compliance of cracks is normalized to the Young modulus) or equivalently $\widetilde{q}=(1-v / 2) \sim 1$ (if the normal compliance is normalized 

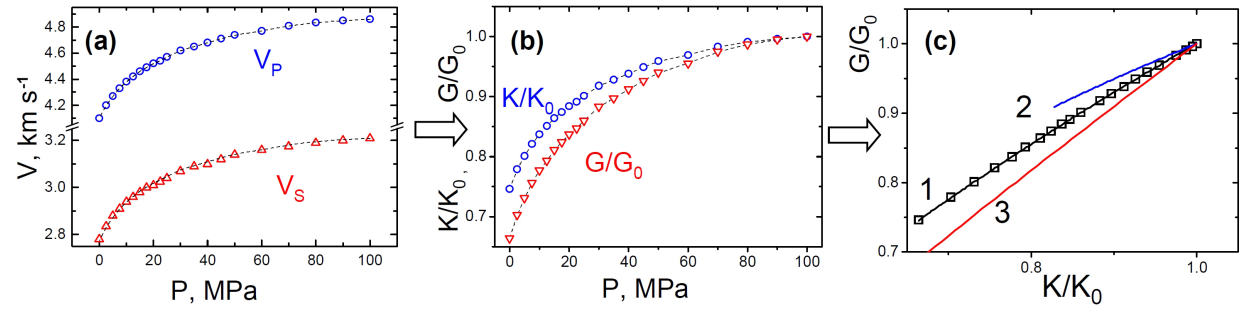

Figure 2. Schematic of determining the $q$-ratio of crack compliances via re-plotting of the P-and S-wave velocities into the trajectory of the point characterizing the rock properties on the $(K, G)$-plane. (a) Initial pressure-dependences of P-and S-wave velocities. (b) Pressure dependences of the normalized bulk and shear moduli derived from the wave velocities. (c) The $(K, G)$-plane representing the normalized moduli plotted one against another and superposed theoretical lines with correctly chosen $q$-ratios (curve 1 ), an about 1.5 times overestimated $q$-ratio (curve 2), and a 1.5 times underestimated $q$-ratio. We emphasize that unknown distributions of cracks over their compliance parameters do affect pressure dependencies but do not affect the $q$-ratio thus estimated (Zaitsev and Sas, 2004; Zaitsev et al., 2017).

to the bulk modulus) (MacBeth, 2004; Sayers and Kachanov, 1995).

Since different effective elastic moduli are sensitive to normal and shear compliances of the compliant defects differently, gradual variation of crack density with pressure should correspond to different trajectories of the point $(K(P), G(P))$ on the $(K, G)$-plane. They are readily expressed via the velocities $V_{P}$ and $V_{\mathrm{s}}$ of the longitudinal compressional wave (P-wave) and shear-wave velocity (Swave), which are routinely measured in experiments. (Certainly a different pair of independent moduli can in principle be used.) Comparing the experimentally obtained trajectory with that theoretically predicted by Eqs. (11)-(14), one can determine the $q$-ratio for real rocks as illustrated in Fig. 2. Such a representation (for example, in the $(K, G)$-plane) allows one to exclude intermediate dependences on pressure that in turn are dependent on the a priori unknown distributions of the cracks over their aspect ratios. The so-plotted single trajectory makes it possible to reduce the freedom in fitting the two initial experimental curves with the additional possibility of scaling pressure axes.

This approach was discussed in detail in Zaitsev and Sas (2004) taking as instructive examples experimental data on pressure dependences (in the range 2-100 MPa) of P- and S-wave velocities for Navajo, Nugget, and Weber sandstones used as examples in Fig. 1 (Coyner, 1984; Mavko and Jizba, 1994). The performed examination showed that only for dry Navajo sandstone was the $q$-ratio (appeared to be $\sim 2.35$ ) more or less consistent with the expectation $q=\left(2-v_{0}\right)\left(1+v_{0}\right) \sim 2.1$ for the model of penny-shaped cracks with free faces, whereas for Nugget sandstone it was about twice as great $(q \sim 4.3)$, and even greater for Weber sandstone $(q \sim 7-8)$. The latter sample even demonstrated a negative Poisson ratio for pressures below $20 \mathrm{MPa}$. For rocks containing compliant inclusions with dominating normal compliance $\left(N_{1}>15 v_{0}+2\left(1+v_{0}\right) N_{2}\right)$, the occurrence of a negative Poisson ratio for cracked rocks does not look surprising (Zaitsev and Sas, 2000) (see also Pasternak and
Dyskin, 2012):

$v_{.}=\frac{v_{0}-\frac{1}{15} N_{\mathrm{n}}+\frac{2}{15}\left(1+v_{0}\right) N_{\mathrm{s}}}{1+\frac{1}{5} N_{\mathrm{n}}+\frac{4}{15}\left(1+v_{0}\right) N_{\mathrm{s}}}$.

However, the Weber sandstone containing cracks with significantly increased normal compliance and high concentration of cracks sufficient for making the Poisson ratio negative looked a rather exotic example. Similar conclusions on the possibility of negative Poisson ratios are known for granular materials, in which inter-grain contacts are characterized by normal compliance significantly dominating over the shear one. However, traditionally, negative Poisson ratios are considered rather exotic cases mostly for various artificial microstructured solids (Gercek, 2007; Lakes, 1993).

In what follows we present results of examination of over 90 rock samples, for which data on pressure dependences of P- and S-wave velocities were taken in Coyner (1984), Freund (1992), and Mavko and Jizba (1994). Figure 4 shows the histogram for the Poisson ratio calculated from the P- and $\mathrm{S}$-wave velocities at the lowest pressure (typically, the available low-pressure data were reported for pressures of several MPa, so that evidently for even lower pressures, crack concentrations were even greater). In this examination we did not try to specially find some specific examples; nevertheless, about $45 \%$ of cracked rocks exhibiting pronounced pressure dependences of the elastic-wave velocities demonstrated negative Poisson ratios in a few (or at least one) lowerpressure points, where the crack concentration was maximal. Typically the lowest pressures were several MPa and maximal pressures were in the range 50-120 MPa.

For those samples, the initial pressure dependences of the $\mathrm{P}$ - and S-wave velocities were re-plotted in the plane of the normalized moduli $(\widetilde{K}, \widetilde{G})$ and the resultant curves were fitted by Eqs. (11) and (12) in order to determine the ratio of the compliances of the cracks assuming that the trajectory can be described by a constant $q$-ratio, $q=N_{1} / N_{2}=$ const. This approximation is not a priori evident at all, but looks fairly reasonable since conventional penny-shaped cracks indeed have the $q$-ratio independent of the aspect ratio and, 
(a)

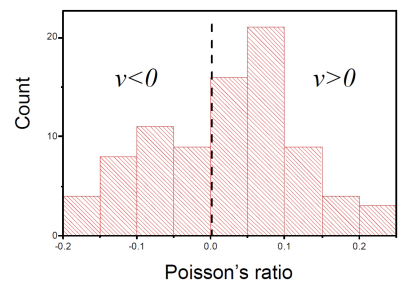

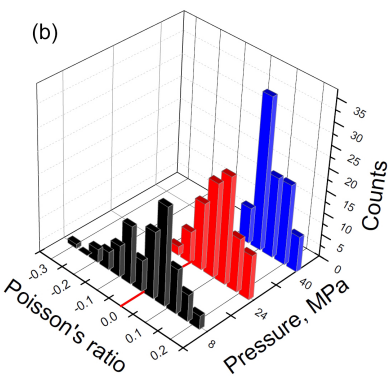

Figure 3. Histograms for the Poisson ratios calculated using P- and S-wave velocities for over 90 rocks (Coyner, 1984; Freund, 1992; Mavko and Jizba, 1994). (a) Data for minimal pressures (mostly about $8 \mathrm{MPa}$ for the available data sets) were used in these calculations. (b) Three histograms for the same set of rocks for pressures $8 \mathrm{MPa}$ (i.e. the same as in panel a), $24 \mathrm{MPa}$, and $40 \mathrm{MPa}$ demonstrating that with increasing pressure the Poisson ratios gradually become positive for all samples due to pressure-induced decrease in crack concentration.

therefore, independent of the pressure of opening/closing of such cracks. For a significant portion of the considered $\sim 90$ rock samples, the pressure-induced variations for the elastic moduli in $(\widetilde{K}, \widetilde{G})$ appeared to be surprisingly well described using the approximation of a constant $q$-ratio.

It was also found that for two tens of samples, the trajectories could be fairly well fitted by a constant $q$-curve at higher pressures, but noticeably deviated at lower pressures, usually exhibiting a trend characteristic for an increasing $q$-ratio. Such deviations occurred for samples with both negative and positive Poisson ratios at low pressures. Therefore, for the moment, in the histograms shown below to characterize the revealed $q$-ratios, we excluded those samples and retained 72 samples with fairly constant $q$-ratios.

Figure 4 shows the histogram for distribution over $q$-ratios among those 72 samples with $q \approx$ const. A striking feature of this histogram is that only the left-most column (only $\sim 20 \%$ of the total number of samples) corresponds to $q \sim 2$ in notations (Zaitsev and Sas, 2000) (or $\widetilde{q} \sim 1$ in notations, MacBeth, 2004; Sayers and Kachanov, 1995), which is typical of penny-shaped cracks and similar conventionally used crack models. Among the 72 samples presented in Fig. 4, almost one-half $(\sim 48 \%)$ exhibits a negative Poisson ratio for maximal crack densities at low pressures.

Figure 5 shows histograms similar to Fig. 4, but separately for 34 samples demonstrating negative Poisson ratios at low pressures and 37 samples with positive Poisson ratios in the entire pressure range. As expected from the abovepresented arguments (see the discussion of Eq. 15), the $q$ ratios for samples with always positive Poisson ratios demonstrate the distribution shifted towards small $q$-ratios (Fig. 5b), whereas for samples with negative Poisson ratios this distribution is clearly shifted towards high $q$-ratios, significantly higher than $q=\left(2-v_{0}\right)\left(1+v_{0}\right) \sim 2$ (or $\left.\widetilde{q} \sim 1\right)$ typical of penny-shaped cracks (Fig. 5a).

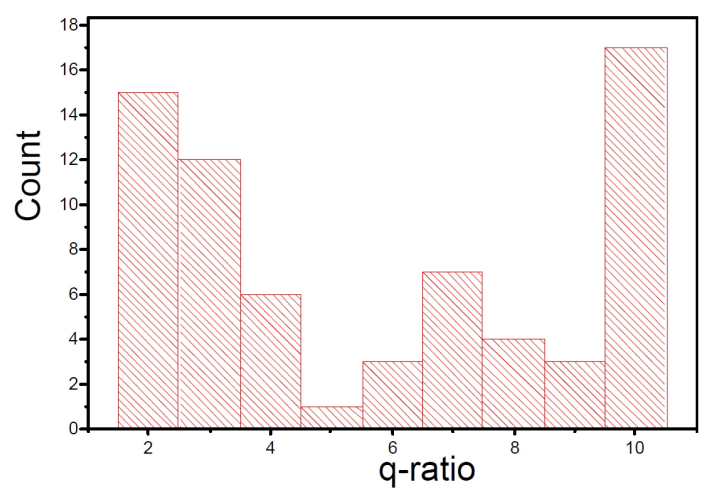

Figure 4. Distribution over $q$-ratios for 72 samples with fairly constant $q$ within the entire pressure ranges including rocks with always positive Poisson ratios together with samples demonstrating negative Poisson ratios at lower pressures. The last column includes all samples with $q \geq 10$.

It can be mentioned that an increased $q$-ratio was also found in the case of samples with always positive Poisson ratios (as can be seen in Fig. 5b). However, applying procedures shown in Fig. 2, we verified that for these samples, the crack density is significantly smaller than for the rocks exhibiting negative Poisson ratios. That is, for rocks with negative Poisson ratios and $q \sim 5-10$ or even greater, typically the crack density is $v_{0}^{(\varsigma)} \sim 1-2$, whereas for rocks with a similarly increased $q$-factor but a positive Poisson ratio even at the lowest pressures, the crack density is significantly lower: $v_{0}^{(5)} \sim 0.1-0.2$.

\section{Conclusions}

In the described examination of pressure-dependent (i.e. nonlinear) elastic rock properties we used approaches from $\mathrm{Za}$ itsev and Sas (2000) and Zaitsev and Sas (2004) and from MacBeth (2004) and Sayers and Kachanov (1995) in which the effective-medium model is based on a generalized phenomenological representation of cracks as highly compliant defects for which their compliance properties are not a priori predetermined, so that the proportion between the normal and shear compliances and their integral amounts can be found from the comparison with experimental data. It should be emphasized that such a comparison is essentially based on the usage of numerous data points obtained in a fairly wide range of pressures. This consideration of the large data sets describing non-linear behaviour of rocks ensures much better reliability and accuracy than comparison of a pair of points (e.g. for two pressure levels). 

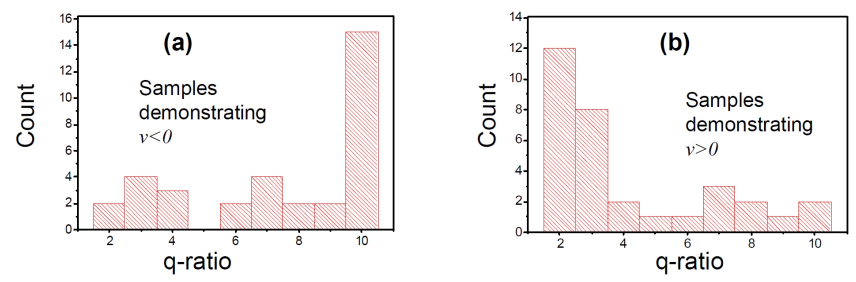

Figure 5. Distributions over $q$-ratios plotted separately for samples from Fig. 4 exhibiting positive and negative Poisson ratios: (a) the histogram for 37 samples with negative Poisson ratios at lower pressures; (b) case of 35 samples with always positive Poisson ratios. The last column in both panels includes all samples with $q \geq 10$.

The performed examination has indicated that properties of compliant cracks in many rocks agree reasonably well with the assumption of uniform distribution $v_{0}^{(\varsigma)} \approx$ const. of the cracks over their compliance parameter (i.e. actually their aspect ratio), which gives a simple way (actually a single parameter $v_{0}^{(\varsigma)}$ ) for comparison of crack concentrations in different samples.

The usage of the theoretical description (Zaitsev and Sas, 2000; MacBeth, 2004; Sayers and Kachanov, 1995; Zaitsev and Sas, 2004) with explicitly introduced normal and shear compliances of the defects made it possible to determine this ratio for real cracks from the trajectory of $(K(P), G(P))$ in the $(K, G)$-plane. Using the literature data on pressure dependences of P- and S-wave velocities (Coyner, 1984; Freund, 1992; Mavko and Jizba, 1994), about 90 rock samples were examined. For a significant $(80 \%)$ portion of the samples the $q$-ratio between the normal and shear compliances appeared to be significantly different from what would be predicted by the penny-shaped crack model. These observations agree with some other results based on smaller volumes of data (Zaitsev and Sas, 2004; Gurevich et al., 2009) that also indicated that quite often conventionally used crack models (like the penny-shaped one) cannot adequately describe properties of real rocks. In fact, for the considered 68 samples that can be well described in the approximation of the constant $q$-ratio, it appears that only $\sim 20 \%$ of rocks demonstrate $q \sim 2$, typical of penny-shaped cracks.

Furthermore, the performed examination of pressure dependences for $\sim 90$ samples (found in the literature without any special selection) revealed that a significant portion of samples (about $45 \%$ ) demonstrated negative Poisson ratios at low pressures, for which concentrations of open cracks were maximal. Discovering such a significant number of naturally auxetic rocks is in contrast to the conventional viewpoint that occurrence of negative Poisson ratios for rocks is an exotic fact (Gercek, 2007; Jizba, 1991). Previously mainly artificial materials with the microstructure engineered to exhibit negative Poisson ratios (auxetic materials) were discussed in the literature (e.g. review Lakes, 1993).
The performed comparison of $q$-ratios has shown that for samples exhibiting negative Poisson ratios, the distribution of determined compliance ratios for cracks shows a clear distortion towards large $q$-ratios (strongly dominating normal compliance of cracks over their shear compliance). This finding perfectly agrees with theoretical models for crack-containing solids and granular materials, according to which negative Poisson ratios can be obtained in nearly isotropic material only if the cracks or contacts have dominating normal compliances (Zaitsev and Sas, 2000; Pasternak and Dyskin, 2012). In contrast, for samples with positive Poisson ratios, the determined distributions of $q$-ratios demonstrated a clear distortion towards small values.

Overall, the obtained results indicate the necessity for further development of crack models to account for the revealed numerous examples of rocks with defects demonstrating $q$ ratios significantly greater than for penny-shaped cracks and similar conventionally used crack models.

Data availability. The authors used experimental data from Coyner (1984), Freund (1992), and Mavko and Jizba (1994).

Competing interests. The authors declare that they have no conflict of interest.

Special issue statement. This article is part of the special issue "Waves in media with pre-existing or emerging inhomogeneities and dissipation". It is a result of the EGU General Assembly 2017, Vienna, Austria, 23-28 April 2017.

Acknowledgement. Vladimir Y. Zaitsev and Andrey V. Radostin acknowledge the support of the Russian Foundation for Basic Research (grant no. 15-05-05143). Arcady V. Dyskin and Elena Pasternak acknowledge the financial support from Australian Research Council linkage project LP 120200797, Australian Worldwide Exploration (AWE) limited, and Norwest Energy NL companies.

Edited by: Sergey Turuntaev

Reviewed by: two anonymous referees

\section{References}

Belyaeva, I. Yu. and Zaitsev, V. Yu.: Nonlinear Elastic Properties of Microinhomogeneous Hierarchically Structured Media, Acoust. Phys., 43, 510-515, 1997.

Belyaeva, I. Yu. and Zaitsev, V. Yu.: The limiting value of the parameter of elastic nonlinearity in structurally inhomogeneous media, Acoust. Phys., 44, 635-640, 1998.

Coyner, K. B.: Effects of stress, pore pressure, and pore fluids on bulk strain, velocity, and permeability in rocks, $\mathrm{PhD}$ thesis, Massachusetts Institute of Technology, 1984. 
Fillinger, L., Zaitsev, V. Y., and Gusev, V.: Nonlinear Relaxational Absorption/Transparency for Acoustic Waves Due to Thermoelastic Effect, Acta Acust. United Ac., 92, 24-34, 2006.

Freund, D.: Ultrasonic compressional and shear velocities in dry clastic rocks as a function of porosity, clay content, and confining pressure, Geophys. J. Int., 108, 125-135, https://doi.org/10.1111/j.1365-246X.1992.tb00843.x, 1992.

Gurevich, B., Makarynska, D., and Pervukhina, M.: Are pennyshaped cracks a good model for compliant porosity?, in: SEG Technical Program Expanded Abstracts, Society of Exploration Geophysicists, 3431-3435, https://doi.org/10.1190/1.3255575, 2009

Guyer, R. A. and Johnson, P. A.: Nonlinear mesoscopic elasticity: the complex behaviour of rocks, soil, concrete, John Wiley \& Sons, 2009.

Han, D.: Effects of porosity and clay content on acoustic properties of sandstones and unconsolidated sediments, $\mathrm{PhD}$ dissertation, Stanford University, 1986.

Jizba, D. L.: Mechanical and acoustical properties of sandstones and shales. PhD Dissertation, Department of Geophysics, Stanford University, 1991.

Lakes, R.: Advances in negative Poisson's ratio materials, Adv. Mater., 5, 293-296, 1993.

Landau, L. D. and Lifshitz, E. M.: Theory of Elasticity, Oxford, Butterworth-Heinemann, https://doi.org/10.1002/adma.19930050416, 1986.

MacBeth, C.: A classification for the pressure-sensitivity properties of a sandstone rock frame, Geophysics, 69, 497-510, https://doi.org/10.1190/1.1707070, 2004.

Mavko, G. and Jizba, D.: The relation between seismic P- and Swave velocity dispersion in saturated rocks, Geophysics, 59, 8792, https://doi.org/10.1190/1.1443537, 1994

Mavko, G. M. and Nur, A.: The effect of nonelliptical cracks on the compressibility of rocks, J. Geophys. Res.-Sol. Ea., 83, 44594468, https://doi.org/10.1029/JB083iB09p04459, 1978.

Morlier, P.: Description de l'etat de fissuration d'une roche a partir d'essais non-destructifs simples, Rock Mech., 3, 125-138, https://doi.org/10.1007/BF01238439, 1971.

Nazarov, V. and Radostin, A.: Nonlinear acoustic waves in microinhomogeneous solids, John Wiley \& Sons, 2015.

O'Connell, R. J. and Budiansky, B.: Seismic velocities in dry and saturated cracked solids, J. Geophys. Res., 79, 5412-5426, https://doi.org/10.1029/JB079i035p05412, 1974.

Pasternak, E. and Dyskin, A. V.: Materials and structures with macroscopic negative Poisson's ratio, Int. J. Eng. Sci., 52, $103-$ 114, https://doi.org/10.1016/j.ijengsci.2011.11.006, 2012.

Gercek, H. Ã.: Poisson's ratio values for rocks, Int. J. Rock Mech. Min., 44, 1-13, https://doi.org/10.1016/j.ijrmms.2006.04.011, 2007.

Sayers, C. M. and Kachanov, M.: Microcrack induced elastic wave anisotropy of brittle rocks, J. Geophys. Res., 100, 4149-4156, https://doi.org/10.1029/94JB03134, 1995.

Sayers, C. M. and Han, D.-H.: The effect of pore fluid on the stressdependent elastic wave velocities in sandstones, in: 72nd Annual International Meeting, SEG, Expanded Abstracts, 1842-1845, https://doi.org/10.1190/1.1817045, 2002.
Trivett, D. H., Pincon, H., and Rogers, P. H.: Investigation of a three-phase medium with a negative parameter of nonlinearity, J. Acoust. Soc. Am., 119, 3610-3617, https://doi.org/10.1121/1.2197804, 2006.

Walsh, J. B.: The effect of cracks in rocks on Poisson's ratio, J. Geophys. Res., 70, 5249-5257, https://doi.org/10.1029/JZ070i020p05249, 1965.

Zaitsev, V. Yu.: A model of anomalous acoustic nonlinearity of micro-inhomogeneous media, Acoust. Lett., 19, 171-176, 1996.

Zaitsev, V. Yu. and Matveev, L. A.: Strain-amplitude dependent dissipation in linearly dissipative and nonlinear elastic microinhomogeneous media, Russ. Geol. Geophys., 47, 694-709, 2006.

Zaitsev, V. Y. and Matveev, L. A.: Giant strain-sensitivity of acoustic energy dissipation in solids containing dry and saturated cracks with wavy interfaces, J. Acoust. Soc. Am., 131, 1-12, https://doi.org/10.1121/1.3664079, 2012.

Zaitsev, V. and Sas, P.: Elastic Moduli and Dissipative Properties of Microinhomogeneous Solids with Isotropically Oriented Defects, Acta Acust. United Ac., 86, 216-228, 2000.

Zaitsev, V. and Sas, P.: Effect of the high-compliant porosity on variations on $\mathrm{P}$-and $\mathrm{S}$-wave velocities in dry and saturated rocks: comparison between theory and experiment, Phys. Mesomech., 7, 37-48, 2004.

Zaitsev, V. Y., Saltykov, V. A., and Matveev, L. A.: Relation between the tidal modulation of seismic noise and the amplitude-dependent loss in rock, Acoust. Phys., 54, 538-544, https://doi.org/10.1134/S1063771008040143, 2008.

Zaitsev, V. Yu., Gurbatov, S. N., and Pronchatov-Rubtsov, N. V.: Nonlinear Acoustic Phenomena in Structure-Heterogeneous Media, Editions of the Institute of Applied Physics RAS, Nizhny Novgorod, 2009a (in Russian).

Zaitsev, V. Y., Dyskin, A., Pasternak, E., and Matveev, L.: Microstructure-induced giant elastic nonlinearity of threshold origin: Mechanism and experimental demonstration, Europhys. Lett., 86, 44005, https://doi.org/10.1209/0295-5075/86/44005, 2009b.

Zaitsev, V. Y., Saltykov, V. A., and Matveev, L. A.: Modulation of high frequency seismic noise by tidal deformations: The features of the phenomenon before strong earthquakes and a probable physical mechanism, Izvestiya, Physics of the Solid Earth, 47, 951-965, https://doi.org/10.1134/S1069351311100156, 2011.

Zaitsev, V. Y., Radostin, A. V., Pasternak, E., and Dyskin, A.: Extracting shear and normal compliances of cracklike defects from pressure dependences of elasticwave velocities, Int. J. Rock Mech. Min., 97, 122-133, https://doi.org/10.1016/j.ijrmms.2017.04.009, 2017.

Zarembo, L. K. and Krasilnikov, V. A.: Nonlinear phenomena in the propagation of elastic waves in solids, Sov. Phys. Uspekhi, 13, 778-797, 1971.

Zimmerman, R. W.: The effect of microcracks on the elastic moduli of brittle materials, J. Mater. Sci. Lett., 4, 1457-1460, https://doi.org/10.1007/BF00721363, 1985. 